\title{
Lattice bosons in a quasi-disordered environment
}

\author{
R. Ramakumar ${ }^{1}$ and A. N. Das ${ }^{2}$ \\ ${ }^{1}$ Department of Physics and Astrophysics, University of Delhi, Delhi-110007, India \\ ${ }^{2}$ Saha Institute of Nuclear Physics, 1/AF Bidhannagar, Kolkata-700064, India
}

(4 May 2013)

\begin{abstract}
In this paper, we study non-interacting bosons in a quasi-disordered one-dimensional optical lattice in a harmonic potential. We consider the case of deterministic quasi-disorder produced by an Aubry-André potential. Using exact diagonalization, we investigate both the zero temperature and the finite temperature properties. We investigate the localization properties by using an entanglement measure. We find that the extreme sensitivity of the localization properties to the number of lattice sites in finite size closed chains disappear in open chains. This feature continues to be present in the presence of a harmonic confining potential. The quasi-disorder is found to strongly reduce the Bose-Einstein condensation temperature and the condensate fraction in open chains. The low temperature thermal depletion rate of the condensate fraction increases considerably with increasing quasi-disorder strength. We also find that the critical quasi-disorder strength required for localization increases with increasing strength of the harmonic potential. Further, we find that the low temperature condensate fraction undergoes a sharp drop to 0.5 in the localization transition region. The temperature dependence of the specific heat is found to be only marginally affected by the quasi-disorder.
\end{abstract}

PACS numbers: 03.75.Hh, 03.75.Lm, 37.10.Jk, 67.85.Hj,72.15.Rn

\section{INTRODUCTION}

The prediction of the localization of Bloch waves moving through a randomly disordered crystal is one of the fundamental results in quantum mechanics ${ }^{1}$. Anderson localization of matter waves is a strong function of the system dimensionality. In two and lower dimensions, all the single particle states are localized by non-zero disorder ${ }^{2,3}$. Anderson localization of matter waves was directly observed in several experiments recently ${ }^{4-7}$. The localization of all the single particles states in one and two dimensions occurs for random disorder (see also the note in Ref. 8). However, if the disorder distribution is deterministic, localization occurs only beyond a critical disorder strength. A particularly simple model in which such a transition from the extended to the localized states occurs is the one dimensional AubryAndré (AA) model ${ }^{9}$. Among the experiments mentioned above, an experiment of particular interest for the purpose of this paper are the studies ${ }^{5}$, by the LENS group, of the localization of a Bose-Einstein condensate of non-interacting lattice bosons subjected to the Aubry-André potential. Our primary motivation comes from these studies in which a finite one-dimensional (1d) Aubry-André model was experimentally realized. There have been extensive theoretical studies of the AA model in the past ${ }^{10-17}$, and in the recent years ${ }^{18-27}$ in response to the experimental developments mentioned above (for recent reviews see Refs. 28-30).

In this paper, our main focus is the finite temperature properties of bosons in finite $1 \mathrm{~d}$ optical lattices with AA quasi-disorder in harmonic confining potentials. Before going to the studies of a Bose condensate in the AA potential in a harmonic trap, we look at the sensitivity of localization properties of a single boson in finite optical lattices with open and closed boundary conditions. We note that the open boundary conditions are more appropriate for the experiments involving bosons in optical lattices. We will show that the extreme sensitivity of the localization properties of a particle in the AA model with closed boundary conditions disappear when open boundary conditions are used. This feature is important when we consider the many-boson system in finite lattices since now one may use any lattice size. Though this continues to be the overall feature after the application of an additional harmonic confining potential, we find a small delocalization tendency before the complete localization transition occurs. This feature results from a competition between the influences of the harmonic potential and the quasi-disorder potential. Further, we go on to study Bose condensation in the AA potential. We study the effects of increasing quasi-disorder on the condensation temperature, condensate fraction, and the specific heat of the system. The quasi-disorder is found to have significant effects on some of these properties. The quasi-disorder strongly reduces the condensation temperature and the condensate fraction near the localization transition. We also find that the fall in the condensate fraction shifts to larger quasi-disorder strengths with increasing harmonic potential strength. Since the focus of this paper is the effects of deterministic quasi-disorder on lattice bosons in harmonic potentials, it is fitting for us to place these studies in the general context of existing work on bosons in disordered environments. In the past, the effects of random disorder on interacting $3 \mathrm{~d}$ continuum bosons have been extensively investigated ${ }^{31-39}$. It has been found that both the 
condensation temperature and the condensate fraction decreases with increasing disorder strength. In the case of noninteracting lattice bosons, the condensation temperature was found ${ }^{40,41}$ to decrease for small filling while showing the opposite trend for large filling. There has been extensive studies ${ }^{42-47}$ on the effects of disorder on interacting lattice bosons within the frame-work of the disordered Bose-Hubbard model. Recent experimental studies ${ }^{48}$ of interacting disordered lattice bosons in a harmonic trap find decreasing condensate fraction with increasing disorder strength.

The rest of this paper is organized as follows. The study of the single particle localization properties in the open and the closed finite length chains is given in the next section (Sec. II). The study on the influence of the quasi-disorder strength on the condensation temperature, condensate fraction, and the specific heat is presented in Sec. III. The conclusions are given in Sec. IV.

\section{THE EFFECTS OF BOUNDARY CONDITIONS ON LOCALIZATION}

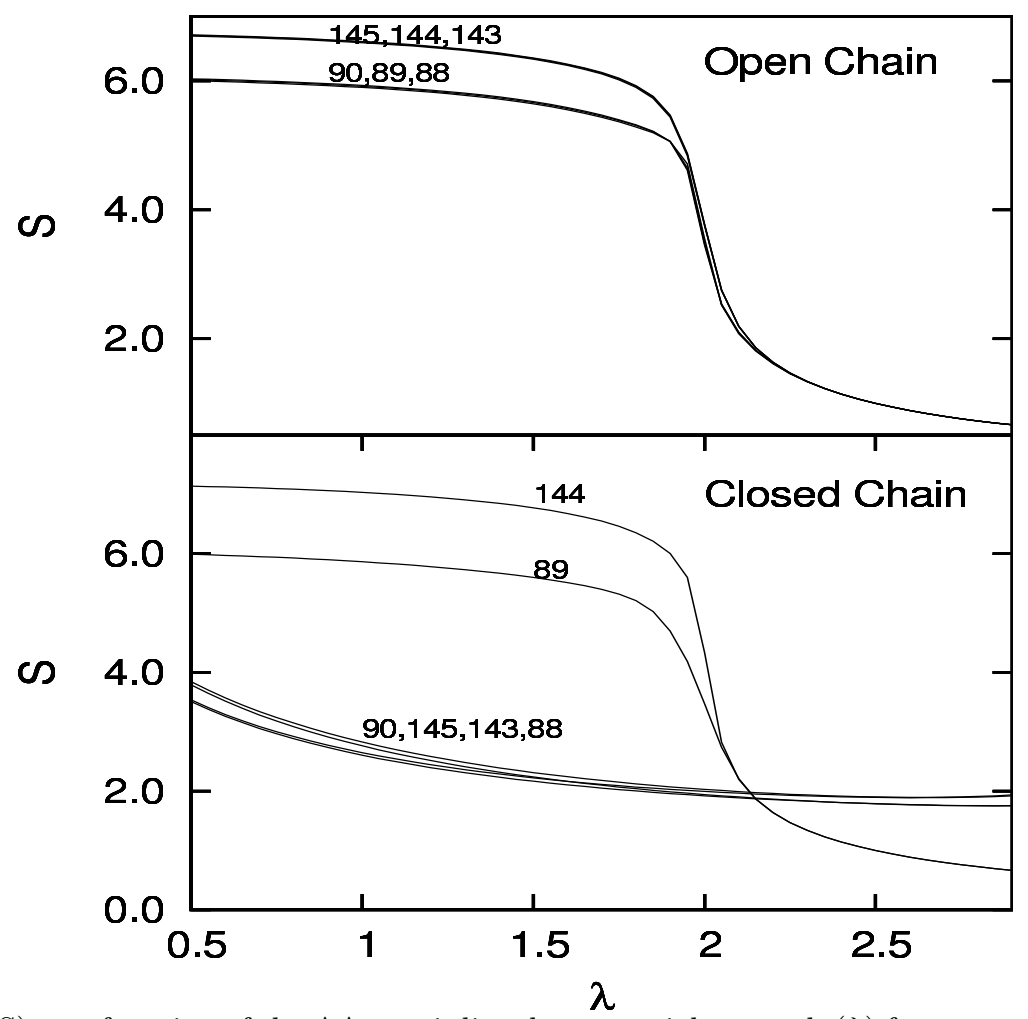

FIG. 1. Entanglement $(S)$ as a function of the AA quasi-disorder potential strength $(\lambda)$ for open and closed chains. Numbers of lattice sites in each chain are shown on the curves.

As stated, we consider harmonically confined lattice bosons with AA disorder potential. The Hamiltonian of this system is:

$$
H=-t \sum_{<i j>}\left(c_{i}^{\dagger} c_{j}+c_{j}^{\dagger} c_{i}\right)+\sum_{i}\left[K a^{2} i^{2}+\lambda \operatorname{Cos}(2 \pi q i)-\mu\right] c_{i}^{\dagger} c_{i}
$$

where $t$ is the kinetic energy gain when a boson hop from site $i$ to its nearest neighbor site $j$ in a one-dimensional optical lattice with a lattice constant $a, c_{i}^{\dagger}$ is a boson creation operator, $K$ the strength of the harmonic potential, $\lambda$ the strength of the AA potential, and $q=(\sqrt{5}+1) / 2$ is the incommensurability parameter. Here $t, K a^{2}$, and $\lambda$ have energy units. All the energies in this paper are measured in units of $t$. After writing this Hamiltonian in a single particle site basis $(|i\rangle)$, we numerically diagonalize it to obtain its eigen-energies and eigen-functions for various lattice sizes, quasi-disorder strengths, and the harmonic potential strengths. The localization properties of the eigen-functions are monitored by calculating the Shannon entropy which measure the quantum entanglement ${ }^{49}$. 
The Shannon entropy is given by

$$
S=-\sum_{i} p_{i} \log _{2} p_{i}
$$

where,

$$
p_{i}=\left|a_{i}\right|^{2}
$$

in which the $a_{i}$ is the $i$ th site amplitude of the ground state wave-function

$$
\left|\psi_{G}\right\rangle=\sum_{i} a_{i}|i\rangle
$$

The quantum entanglement is maximum for a fully extended state and is zero for a state localized on a site.

The results of our calculations for open and closed chains ${ }^{50}$, in the absence of the harmonic potential, are shown in Fig. 1. In the (finite size) closed chain results shown, the entanglement $(S)$ falls abruptly at $\lambda \approx 2$ signifying a transition from an extended state to a localized state for lattices with 144 and 89 sites, but not for other numbers of lattice sites as shown in Fig. 1. These numbers (89 and 144) are members of the Fibonacci series, and the ratios between two consecutive Fibonacci numbers approaches $q=(\sqrt{5}+1) / 2$, the golden ratio, in the AA potential in the large system limit. As has been noted earlier ${ }^{13,15,24}$, the transition occurs for these numbers because of matching of the periodic boundary conditions on the lattice and the quasi periodicity of the AA potential. This extreme sensitivity disappears in the chains with open boundary conditions, as shown in the top panel of the Fig. 1. The effect of the harmonic potential on the localization for various values of $k=K a^{2}$ is shown in Fig. 2 . It is clear that $S$ continues to maintain the overall feature of the $k=0$ case. However, we find a small increase in $S$ before the complete localization transition occurs. This can be understood by examining the nature of the wave-functions shown in Fig. 3 . We have plotted the square of

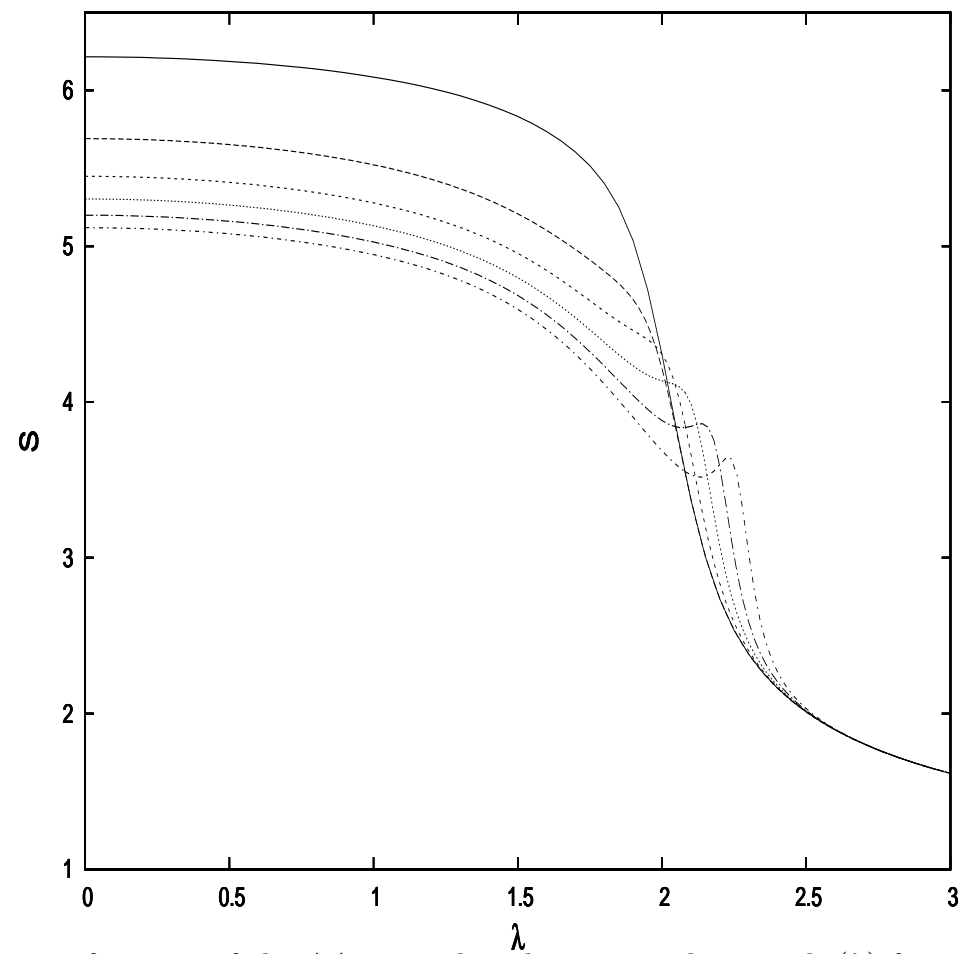

FIG. 2. Entanglement $(\mathrm{S})$ as a function of the AA quasi-disorder potential strength $(\lambda)$ for an open chain with 100 lattice sites in harmonic potentials of different strengths. The curves shown are for : $\mathrm{k}=0$ (solid), 0.00001 (long dash), 0.00002 (short dash), 0.00003 (dot), 0.00004 (long dash-dot), and 0.00005 (short dash-dot). The $k$ is given in units of $t$. 




FIG. 3. The wave functions for $\mathrm{k}=0.00005$.

the amplitude of the ground state wave function as a function of $i$ for 100 sites with $k=0.00005$ and for different values of $\lambda \geq 1$. For this case, the $a_{i}^{2}$ exhibit two peaks around $i=46$ and 54 . The heights of these two peaks increase with increasing $\lambda$ up to $\lambda=2.15$. The entanglement, correspondingly, decreases up to $\lambda=2.15$ for $k=0.00005$. As $\lambda$ increases further, the peak heights at $i=46$ and 54 positions decrease, while another two peaks appear around $i=33$ and 67 . For $\lambda$ around 2.24 the peak heights at these four positions become almost equal. On further increase in $\lambda$, the peak heights at $i=46$ and 54 decrease while those at $i=33$ and 67 increase rapidly. We find that the $S$ shows a maximum around 2.24 and then decreases rapidly. It is clear that the small increase in $S$ results from a competition between the tendency of the quasi-disorder potential to localize the wave function on two sets of lattice sites away from the center and tendency of the harmonic potential to bring the particle to the center of the trap. This section dealt with the single boson properties. In the next section we consider the many-boson system.

\section{BOSE CONDENSATION IN THE AA POTENTIAL}

In this section we study the Bose condensation temperature, the temperature dependence of the condensate fraction and the specific heat of harmonically confined lattice bosons in AA potentials ${ }^{51}$. To study these properties for a finite system, we first numerically diagonalize the Hamiltonian matrix to obtain the single boson energy levels $E_{i}$. In terms of $E_{i}$, the total number of bosons is given by the number equation

$$
N=\sum_{i=0}^{m} N\left(E_{i}\right)
$$

where $E_{0}$ and $E_{m}$ are the lowest and the highest single boson energy levels, and

$$
N\left(E_{i}\right)=\frac{1}{e^{\beta\left(E_{i}-\mu\right)}-1}
$$

in which $\beta=1 / k_{B} T$ with $k_{B}$ the Boltzmann constant and $T$ the temperature. The chemical potential and boson populations in the various energy levels are calculated using the boson number equation. The specific heat is calculated from the temperature derivative of total energy

$$
E_{t o t}=\sum_{i=0}^{m} N\left(E_{i}\right) E_{i}
$$


and the condensation temperature ${ }^{52,53}\left(T_{0}\right)$ is determined by solving the number equation after setting $N_{0}=0$ and $\mu=E_{0}$. The isothermal compressibility $\left(\kappa_{T}\right)$ of non-interacting bosons is given by ${ }^{54}$

$$
\begin{aligned}
\kappa_{T} & =\frac{1}{N} \frac{\delta N}{\delta \mu} \\
& =\beta\left[1+\frac{\sum_{i=0}^{m}\left(N\left(E_{i}\right)\right)^{2}}{\sum_{i=0}^{m} N\left(E_{i}\right)}\right] .
\end{aligned}
$$

We have calculated the isothermal compressibility for our system using Eq. (9) and studied its variation with temperature for different values of the AA potential strength $(\lambda)$.

The effect of increasing quasi-disorder strength on the condensation temperature is shown in Fig. 4. The

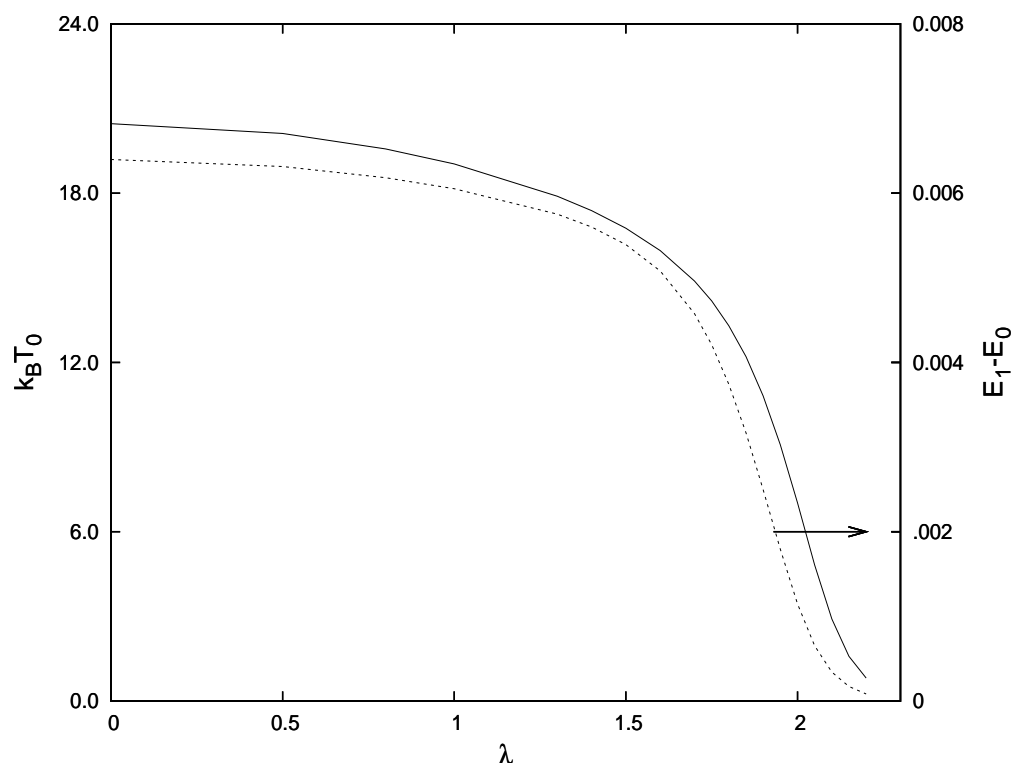

FIG. 4. The condensation temperature $\left(\mathrm{T}_{0}\right)$ as a function of $\lambda$ (solid line) for 10000 bosons in a $1 \mathrm{~d}$ lattice of 100 sites. The harmonic trap strength $\mathrm{k}=0.00001$. The dashed line shows the variation of the difference between the energies of the first excited state $\left(\mathrm{E}_{1}\right)$ and the ground state $\left(\mathrm{E}_{0}\right)$. 


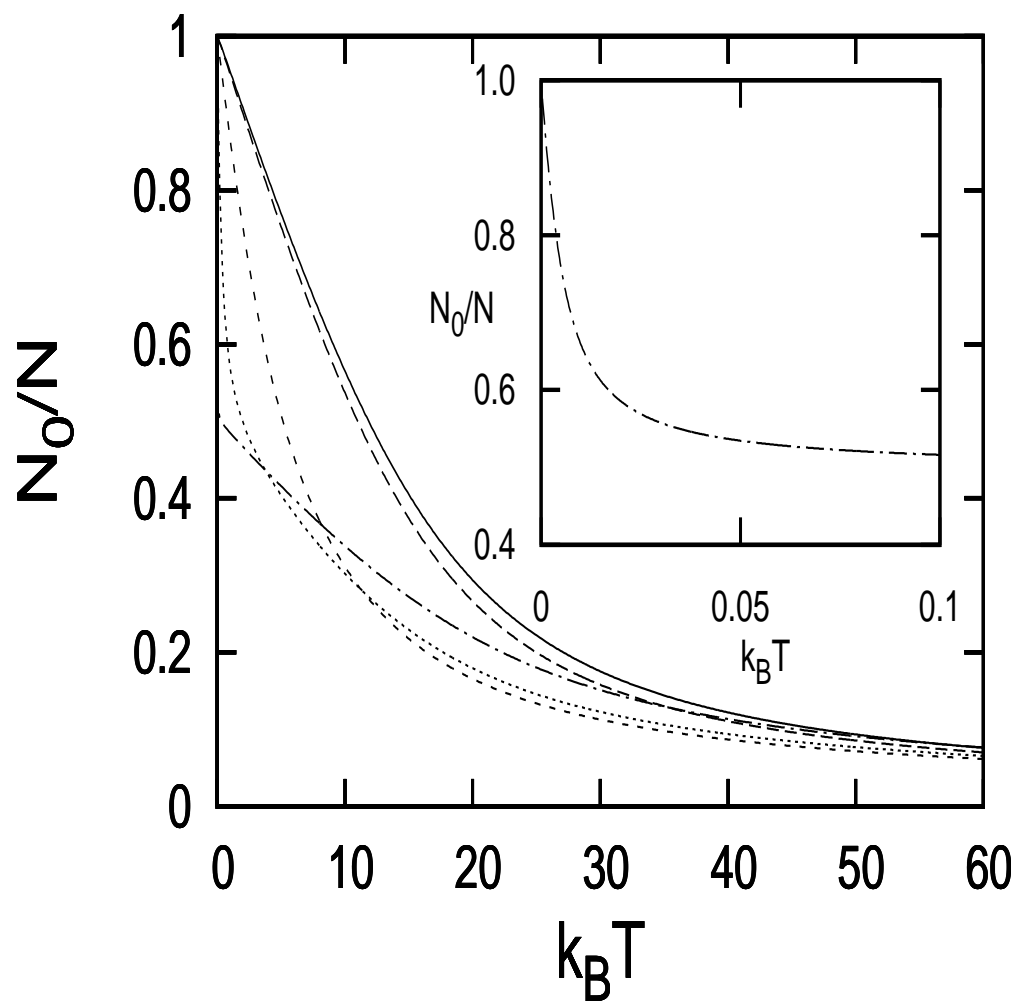

FIG. 5. The temperature dependence of the condensate fraction for different values of $\lambda$ for 10000 bosons in a lattice of 100 sites in a harmonic potential of strength $\mathrm{k}=0.00001$. The results shown are for : $\lambda=0$ (solid line), 1.0 (long dash), 2.0 (short dash), 2.2 (dot), and 2.5 (dash-dot). The inset shows the low temperature variation for $\lambda=2.5$.

quasi-disorder clearly leads to a suppression of $T_{0}$. The maximum reduction occurs in the transition region between the extended and the localized states. We also find an interesting connection between the variation of the $T_{0}$ and the quasi-disorder dependence of the energy difference $\left(E_{1}-E_{0}\right)$ between the first excited state and the ground state. The $T_{0}$ is found to closely track $\left(E_{1}-E_{0}\right)$. The reduction of $\left(E_{1}-E_{0}\right)$ leads to an increase in the one-boson density of energy states at the bottom of the energy spectrum. This leads to the reduction in $T_{0}$. The temperature dependence of the ground state occupancy with various quasi-disorder strengths is shown in Fig. 5. With increasing quasi-disorder strength, the thermal depletion rate is found to increase considerably at low temperatures. This is especially significant as one moves in to the localization transition region. In the localized limit, the condensate fraction decreases sharply with a slight increase in temperature as is clear from the results for $\lambda=2.5$. 


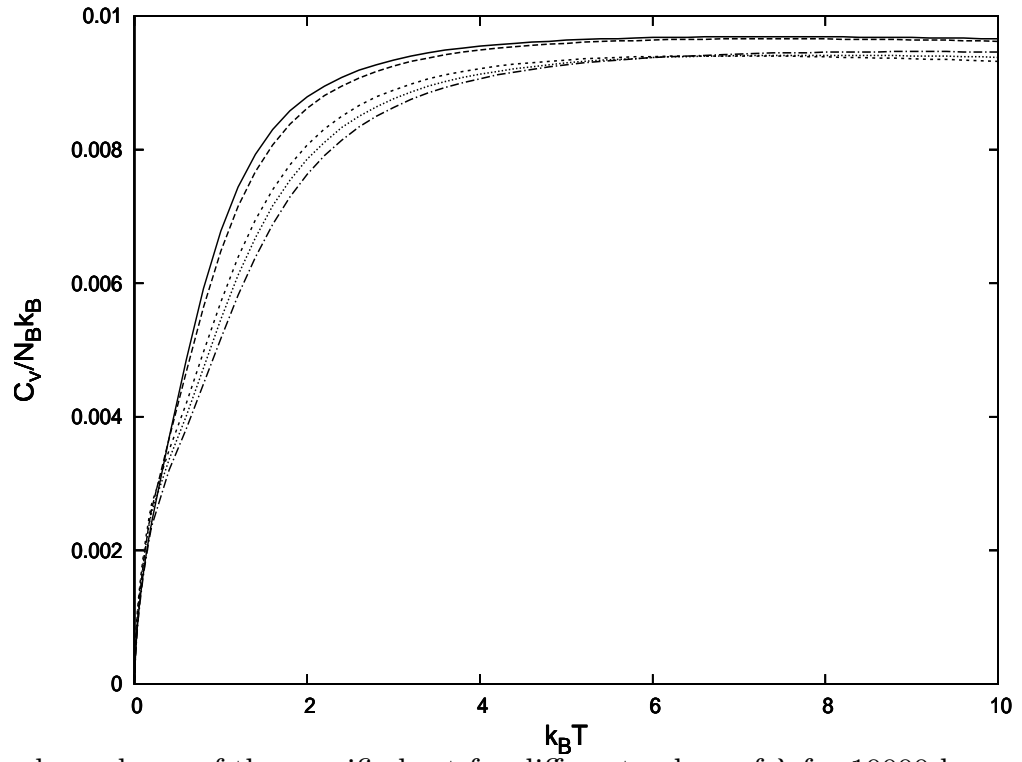

FIG. 6. The temperature dependence of the specific heat for different values of $\lambda$ for 10000 bosons in a lattice of 100 sites in a harmonic potential of strength $\mathrm{k}=0.00001$. The results shown are for : $\lambda=0$ (solid line), 1.0 (long dash), 2.0 (short dash), 2.2 (dot), and 2.5 (dash-dot).

The temperature dependence of the specific heat for various values of the quasi-disorder strength is shown in Fig. 6. Even though in the range of quasi-disorder strengths shown, the system has undergone the localization transition, the specific heat is found to be only marginally affected by the quasi-disorder.

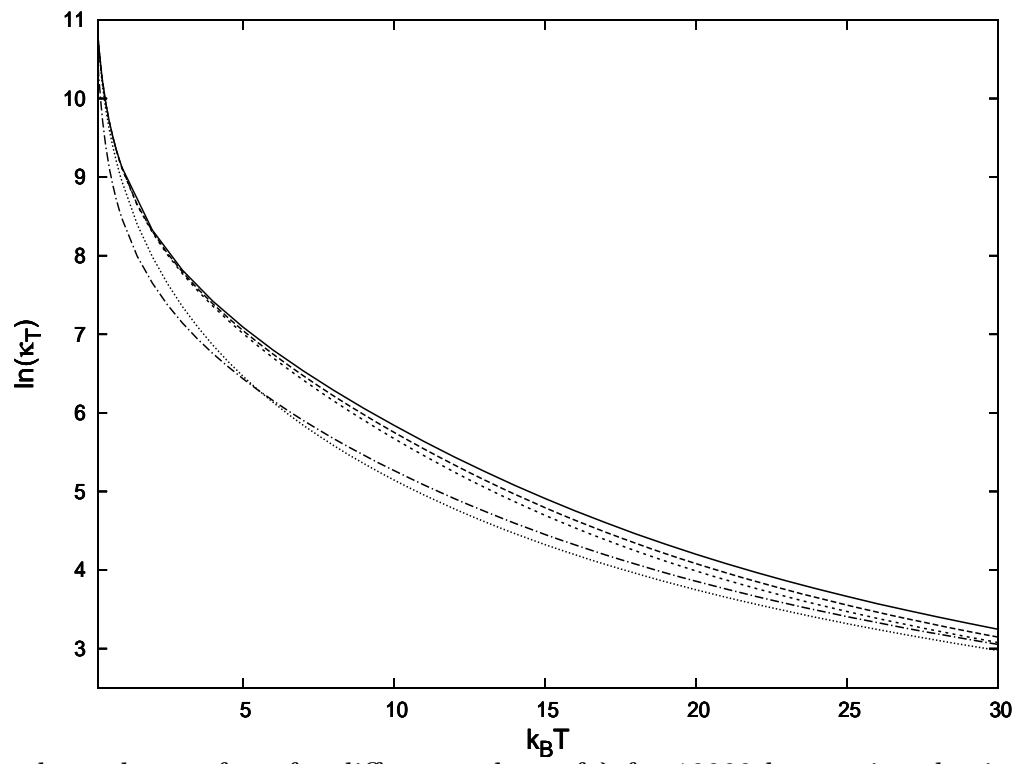

FIG. 7. The temperature dependence of $\kappa_{T}$ for different values of $\lambda$ for 10000 bosons in a lattice of 100 sites in a harmonic potential of strength $\mathrm{k}=0.00001$. The results shown are for : $\lambda=0$ (solid line), 1.0 (long dash), 1.3 (short dash), 2.0 (dot), and 2.2 (dash-dot).

In Fig. 7 we showed the variation of the isothermal compressibility with temperature for different $\lambda$ values. The compressibility increases sharply with decreasing temperature as expected from its expression in Eq. (9), since both the $1 / T$ and $\sum\left(N\left(E_{i}\right)\right)^{2} / N$ terms in $\kappa_{T}$ increase with decreasing temperature. Regarding $\lambda$ dependence of $\kappa_{T}$, we find that at high temperatures it decreases with increasing $\lambda$ up to $\lambda=2$, beyond which, as the system enters into the localized regime, $\kappa_{T}$ increases with increasing $\lambda$. At low temperatures $\kappa_{T}$ decreases with $\lambda$ even beyond $\lambda=2$. 
This behavior is similar to that observed for $N_{0} / N$ in Fig. 5 . It may be mentioned that the term $\sum\left(N\left(E_{i}\right)\right)^{2} / N$ in $\kappa_{T}$ is mainly determined by its ground state contribution, i.e. $N_{0}^{2} / N$, when $N_{0}$ reaches a macroscopic value.

The effect of increasing quasi-disorder strength on the thermal depletion of the condensate fraction was shown in Fig. 5. We now go on to a detailed investigation of the quasi-disorder induced depletion of the condensate.

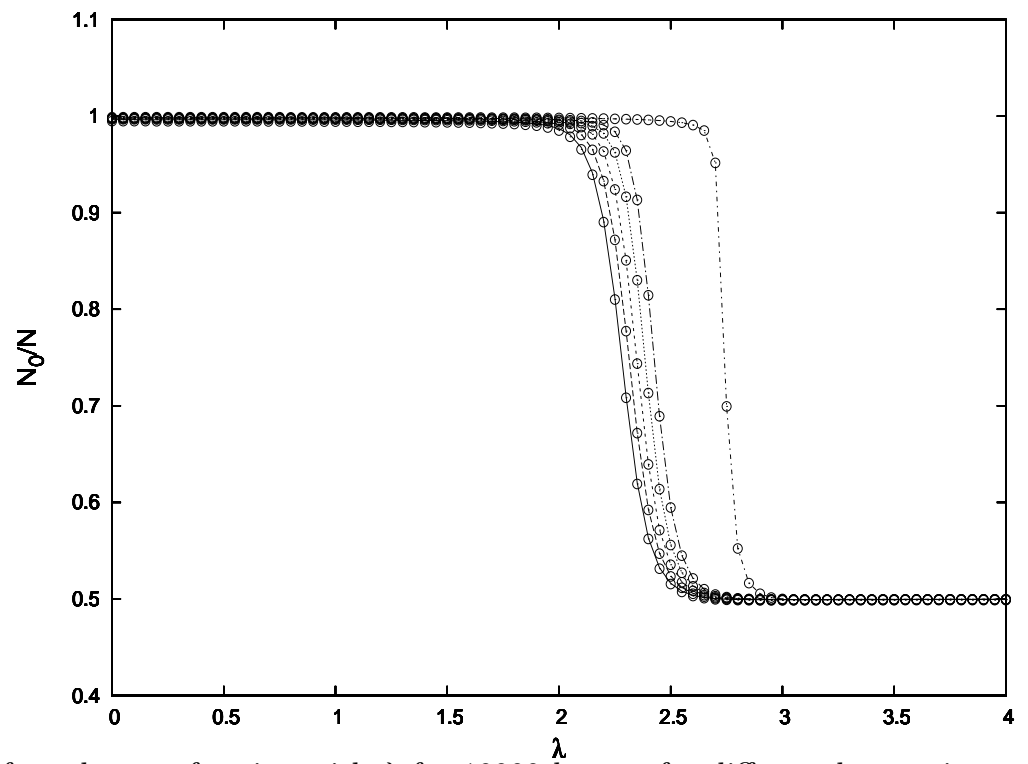

FIG. 8. The variation of condensate fraction with $\lambda$ for 10000 bosons for different harmonic potential strengths. The lines are for 100 sites for k values: 0.00001 (solid), 0.00002 (long-dash), 0.00003 (short-dash), 0.00004 (dot), 0.00005 (long dash-dot), and 0.0001 (short dash-dot). The circles are for 300 sites for the same $\mathrm{k}$ values. The temperature is $\mathrm{k}_{B} \mathrm{~T}=0.1$.

In Fig. 8, we present the variation of the condensate fraction with quasi-disorder strength for different harmonic potentials. We find that the increase in quasi-disorder strength leads to two major effects. The first one is the sharp drop in the condensate fraction to 0.5 in the localization transition region and second is the shift of this fall to higher values of quasi-disorder strengths with increasing strength of the harmonic confinement. The latter result correlates well with the localization properties given in Fig. 2. This shift is due to larger quasi-disorder strengths required for the localization transition when the harmonic potential increases. In order to understand the first effect, we examined the energy level differences between the ground state and the lower excited energy levels. In Fig. 9, we have shown the energy level difference, as a function of quasi-disorder strength, between the first excited state and the ground state, and between the second excited state and the ground state. When the energy difference between the first excited state and the ground state becomes very very small, both the states are almost equally populated, and $N_{0} / N$ reduces to about 0.5. Note that the energy difference between the first excited and the ground state remains finite, though small, even for large values of $\lambda$, which is not visible in the scale of this figure. Note also that that the $E_{2}-E_{0}$ shows a minimum in the vicinity of the localization transition and then increases fast as the system moves deeper in to the localized regime. 


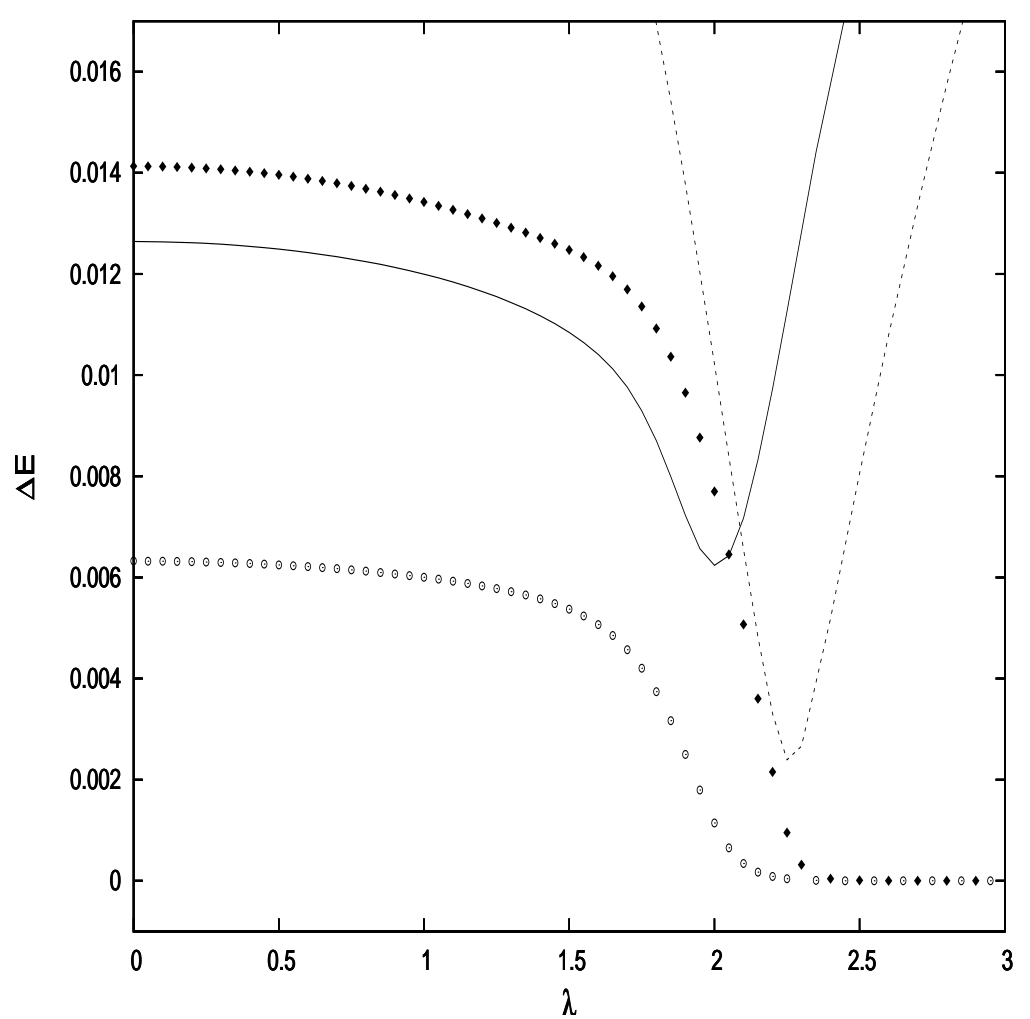

FIG. 9. The variation of the energy difference $\Delta \mathrm{E}$ between low lying levels with $\lambda$. The results are for $\mathrm{k}=0.00001: E_{1}-E_{0}$ (open circles) and $E_{2}-E_{0}$ (solid line) and for $\mathrm{k}=0.00005: E_{1}-E_{0}$ (filled diamonds) and $E_{2}-E_{0}$ (dash line).

\section{CONCLUSIONS}

In this paper, we investigated some properties of a single boson as well as many boson systems in finite onedimensional optical lattices with Aubry-Andre quasi-disorder in harmonic confinements. We find that the open lattices do not show the extreme sensitivity of the localization properties seen in finite closed lattices. This continues to be the case in the presence of an overall harmonic confining potential. In the presence of the harmonic potential, we find a small delocalization tendency before a completely localized state is reached. On the basis of the quasidisorder induced changes occurring in the single particle wave-function, we argued that this tendency is due to a competition between the effects of the harmonic potential and the quasi-disorder potential. For the many-boson system, we studied the effects of quasi-disorder on the condensation temperature, the condensate fraction, and the specific heat. We find that the condensation temperature decreases with increasing quasi-disorder strength, the rate of the reduction is maximum in the localization transition region. The quasi-disorder is found to have a significant effect on the condensate fraction. With increasing quasi-disorder, the thermal depletion rate is found to increase considerably, especially in the localization transition region. In the localized limit, the condensate fraction decreases significantly with a small increase in the temperature. We also studied the variation of the low temperature condensate fraction with increasing quasi-disorder strength. We find that the condensate fraction shows a sharp drop to 0.5 in the localization transition region. We also find that this fall shifts to higher quasi-disorder strengths with increasing strength of the harmonic confining potential. The effect of quasi-disorder on the specific heat is found to be a marginal reduction in a certain range of temperature.

\section{ACKNOWLEDGMENTS}

RRK thanks Professor Milan Sanyal, Director, SINP and Professor Bikas Chakrabarti, Head, TCMP Division, SINP for hospitality at SINP. RRK also thanks Professor Laurent Sanchez-Palencia (LCF, Palaiseau) for drawing his attention to two recent papers from his group. 
${ }^{1}$ P. W. Anderson, Phys. Rev. 109, 1492 (1958).

${ }^{2}$ N. F. Mott and W. D. Twose, Adv. Phys. 10, 107 (1961).

${ }^{3}$ E. Abrahams, P. W. Anderson, D. C. Licciardello, and T. V. Ramakrishnan, Phys. Rev. Lett. 42, 673 (1979).

${ }^{4}$ J. Billy, V. Josse, Z. Zuo, A. Bernard, B. Hambrecht, P. Lugan, D. Clément, L. Sanchez-Palencia, P. Bouyer, and A. Aspect, Nature 453, 891(2008).

${ }^{5}$ G. Roati, C. D'Errico, L. Fallani, M. Fattori, C. Fort, M. Zaccanti, G. Modugno, M. Modugno, and M. Inguscio, Nature 453, 895 (2008).

${ }^{6}$ S. S. Kondov, W. R. McGehee, J. J. Zirbel, and B. DeMarco, Science 334, 66 (2011).

${ }^{7}$ F. Jendrzejewski, A. Bernard, K. Müller, P. Cheinet, V. Josse, M. Piraud, L. Pezzé, L. Sanchez-Palencia, A. Aspect, and P. Bouyer, Nature Phys. 8, 398 (2012). See also M. Piraud, L. Pezzeé, and L. Sanchez-Palencia, Europhys. Lett. 99, 50003 (2012).

${ }^{8}$ For an investigation into the changes produced by the trapping potential, which obtains in cold atom experiments, on Anderson localization in one and two dimensions, see L. Pezzé and L. Sanchez-Palencia, Phys. Rev. Lett. 106, 040601 (2011).

${ }^{9}$ S. Aubry and G. Andŕe, Ann. Isr. Phys. Soc. 3, 133 (1980).

${ }^{10}$ M. Ya Azbel, Phys. Rev. Lett. 43, 1954 (1982).

${ }^{11}$ I. M. Suslov, Zh. Éksp. Teor. Fiz. 83, 1079 (1982) [Sov. Phys. JETP 56, 612 (1982)].

12 D. J. Thouless, Phys. Rev. Lett. 28, 4272 (1983).

${ }^{13}$ M. Kohmoto, Phys. Rev. Lett. 51, 1198 (1983).

${ }^{14}$ M. Kohmoto, L. P. Kadanoff, and C. Tang, Phys. Rev. Lett. 50, 1870 (1983).

${ }^{15}$ G.-L. Ingold, A. Wobst, C. Aulbach, and P. Hanggi, Eur. Phys. J. B 30, 175 (2002).

${ }^{16}$ C. Aulbach, A. Wobst, G.-L. Inglold, P. Hänggi, and I. Varga, New J. Phys. 6, 70 (2004).

${ }^{17}$ D. J. Boers, B. Goedeke, D. Hinrichs, and M. Holthaus, Phys. Rev. A 75, 063404 (2007).

${ }^{18}$ G. Roux, T. Barthel, I. P. McCulloch, C. Kollath, U. Schollwöck, and T. Giamarchi, Phys. Rev. A 78, 023628 (2008).

${ }^{19}$ X. Deng, R. Citro, A. Minguzzi, and E. Orignac, Phys. Rev. A 78, 013625 (2008).

20 M. Modugno, New J. Phys. 11, 033023 (2009).

${ }^{21}$ M. Larcher, F. Dalfovo, and M. Modugno, Phys. Rev. A 80, 053606 (2009).

${ }^{22}$ S. K. Adhikari and L. Salasnich, Phys. Rev. A 80, 023606 (2009).

${ }^{23}$ X. Deng, R. Citro, E. Orignac, and A. Minguzzi, Eur. Phys. J. B 68, 435 (2009).

24 J. C. C. Cestari, A. Foerster, and M. A. Cusmaõ, Phys. Rev. A 82, 063634 (2010).

${ }^{25}$ M. Albert and P. Leboeuf, Phys. Rev. A 81, 013614 (2010). This paper contains a detailed study of the similarities and the differences between the localization in Anderson model and the AA model. See also M. Piraud, A. Aspect, and L. Sanchez-Palencia, Phys. Rev. A 85, 063611 (2012).

${ }^{26}$ M. Larcher, M. Modugno, and F. Dalfovo, Phys. Rev. A 83, 013624 (2011).

${ }^{27}$ J. Biddle, D. J. Priour,Jr., B. Wang, and S. Das Sarma, Phys. Rev. B 83, 075105 (2011).

${ }^{28}$ G. Modugno, Rep. Prog. Phys. 73, 102401 (2010).

${ }^{29}$ L. Sanchez-Palencia and M. Lewenstein, Nature Phys. 6, 87 (2010).

${ }^{30}$ B. Shapiro, J. Phys. A 45, 143001 (2012).

${ }^{31}$ K. Haung and H. F. Meng, Phys. Rev. Lett. 69, 644 (1992).

32 S. Giorgini, L. Pitaevskii, and S. Stringari, Phys. Rev. B 49, 12938 (1994).

33 A. V. Lopatin and V. M. Vinokur, Phys. Rev. Lett. 88, 235503 (2002).

${ }^{34}$ G. E. Astrakharchik, J. Boronat, J. Casulleras, and S. Giorgini, Phys. Rev. A 66, 023603 (2002).

${ }^{35}$ M. Kobayashi and M. Tsubota, Phys. Rev. B 66, 174516 (2002).

${ }^{36}$ V. I. Yukalov, E. P. Yukalova, K. V. Krutitsky, and R. Graham, Phys. Rev. A 76, 053623 (2007).

${ }^{37}$ V. I. Yukalov and R. Graham, Phys. Rev. A 75, 023619 (2007).

${ }^{38}$ G. M. Falco, A. Pelster, and R. Graham, Phys. Rev. A 75, 063619 (2007).

${ }^{39}$ S. Pilati, S. Giorgini, M. Modugno, and N. Prokof'ev, New J. Phys. 12, 073003 (2010).

${ }^{40}$ L. Dell'Anna, S. Fantoni, P. Sodano, and A. Trombettoni, J. Stat. Mech. 11012 (2008).

${ }^{41}$ L. Dell'Anna, S. Fantoni, P. Sodano, and A. Trombettoni, Laser Phys. 19, 571 (2009).

42 There has been intensive studies of the disordered Bose-Hubbard model since the late eighties (Ref. 43). Since we are not considering interacting bosons in this paper, we request the reader to consult the recent works (Refs. 44-47), and the references contained therein.

${ }^{43}$ M. P. A. Fisher, P. B. Weichman, G. Grinstein, and D. S. Fisher, Phys. Rev. B 40, 546 (1989).

${ }^{44}$ S. G. Söyler, M. Kiselev, N. V. Prokof'ev, and B. V. Svistunov, Phys. Rev. Lett. 107, 185301 (2011).

${ }^{45}$ F. Lin, E. S. Srensen, and D. M. Ceperley, Phys. Rev. B 84, 094507 (2011). 
${ }^{46}$ P. Pisarski, R. M. Jones, and R. J. Gooding, Phys. Rev. A 83, 053608 (2011).

${ }^{47}$ F. Krüger, S. Hong, and P. Phillips, Phys. Rev. B 84, 115118 (2011).

${ }^{48}$ M. White, M. Pasienski, D. McKay, S. Q. Zhou, D. Ceperley, and B. DeMarco, Phys. Rev. Lett. 102, 055301 (2009).

49 J. -M. Stéphan, S. Furukawa, G. Misguich, and V. Pasquier, Phys. Rev. B 80, 184421 (2009).

${ }^{50}$ In the numerical calculations, the open-chain and the closed chain results are obtained by taking $t_{N_{s}, 1}=t_{1, N_{s}}=0$ and $t_{N_{s}, 1}=t_{1, N_{s}}=t$, respectively, where $N_{s}$ is the number of sites.

${ }^{51}$ Since our system is one-dimensional, one has a quasi-condensate at finite temperatures.

${ }^{52}$ W. Ketterle and N. J. van Druten, Phys. Rev. A 54, 656 (1996).

${ }^{53}$ R. Ramakumar, A. N. Das, and S. Sil, Eur. Phys. J. D 42, 309 (2007).

${ }^{54}$ M. Krämer, C. Menotti, L. Pitaevskii, and S. Stringari, Eur. Phys. J. D 27, 247 (2003). 